\title{
أثر بعض المثغيرات الاقتصادية في عرض النقد الواسع لدول الاتحاد النقدي الاوريي
}

\author{
أ.م.د زاهد قاسم بدن الساعدي، كلية الادارة والاقتصاد- جامعة ميسان، العراق \\ أ. م.د. غسان ابراهيم احمد، كلية الادارة والاقتصاد -جامعة نوروز، أقليم كوردستان العراق
}

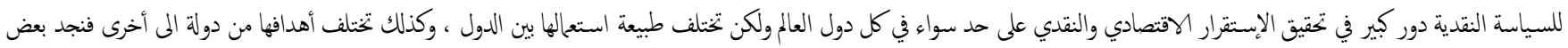

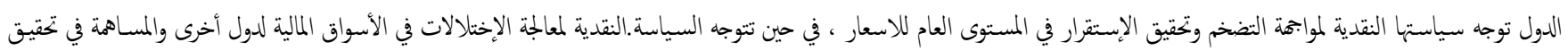

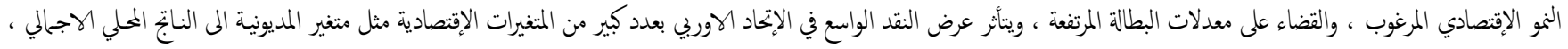

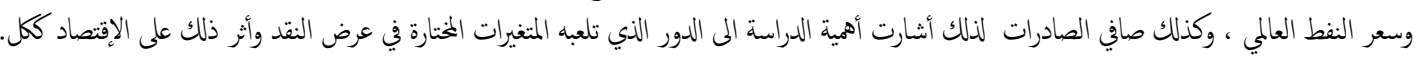

الكلمات المفتاحية: اداة عرض النقد ، الاتحاد النقدي الووربي ، الدين.

1

يأخذ البحث أهميته من أداة عرض النقد وأثر بعض متغيرات الاقتصاد الكلي عليها لاسيا الاسعار العالمية للنفط والمديونية وحالة ميزان المدفوعات وتأثير ذلك على نمو الناتج المحلي الإجالي لدول الإتحاد النقدي الاوربي بشكل عام.

2.1

تعد دول الاتحاد الأوربي ذات سياسة نقدية موهدة يرسمها البنك المركزي الأوربي وتنفذ من قبل بنوك مركزية في هذه الدول إلا ان اداة عرض النقد تواجه عدة معوقات منها السياسة المالية المتعددة في هذه الدول اضافة الى اثارها التضخمية ، لذلك تكمن المشكلة في كيفة تكمل هذه السياسات والأهداف مع بعضها البعض باستخدام أداة عرض النقد للسياسة النقدية الموحدة.

\section{1 أهداف البحث}

- دراسة مفاهيم إدارة السياسة النقدية في دول الاتحاد الاوربي . - م - النعرف على طبيعة عمل البنك المركزي الووربي.

- دراسة أثر بعض المثغيرات الاقتصادية في اداة عرض النقد لدول الاتحاد

$$
\text { النقدي الاوربي. }
$$

- متليل وقياس لأثر المتغيرات الاقتصادية في اداة عرض النقد في دول

$$
\text { الاتحاد النقدي الاوربي. }
$$

\section{1}

ينطلق البحث من الفرضية التالية ان البنك المركزي الاوربي يمارس دور ضعيفاً
يعد الاتحاد الاقتصادي والنقدي الاوربي مشروع تاريخي يدير سياسته النقدية بنك مركزي واحد متمثل بالبنك المركزي الووربي (ECB) ، تم منحه بمحوعة من المهام كإصدار العملة (اليورو ) ، وتطبيق السياسة النقدية وإدارة الإحتياطيات الأجنبية والتشغيل الإنسيابي لأنظمة الدف. يتألف النظام الأوروبي للبنوك المركزية من البنك المركزي الأوروبي والبنوك المركزية الوطنية للدول الأعضاء (ESCB) في الاتحاد النقدي الأوروبي. الهدف الأساسي من النظام الاوربي للبنوك المركزية (ESCB ) الأوروبي في قراراته على أساس إستراتيجية السياسة النقدية المكونة من تدابير السياسة النقدية القياسية وغير القياسية. وللسياسة النقدية الموحدة للبنك المركزي الأوروبي أدواته الرئيسية المتمثلة بعمليات السوق المفتوحة ، التسهيلات الدائمة والإحتفاظ بالحد الأدنى من الإحتياطيات. ، قام البنك المركزي الأوروبي أيضًا بتغيير إستراتيجيته في مجال الاتصالات من خلال تقديم توجيهات مستقبلية بشأن المسار المستقبلي لسياسة سعر الفائدة للبنك المركزي الأوروبي ، بشرط النظر في استقرار الأسعار واتخذ عددًا من تدابير السياسة النقدية القياسية المتثثلة بشراء الأصول والسندات السيادية في السوق الثانوية ، بهدف حاية إستقرار الأسعار وفعالية آلية نقل السياسة النقدية إستجابةً للأزمة المالية.

يستمد البحث أهميته من أثر بعض المتغيرات الاقتصادية المتمثلة بالمديونية وسعر النفط العالمي وصافي الميزان التجاري في عرض النقد الواسع لجول الاتحاد النقدي 


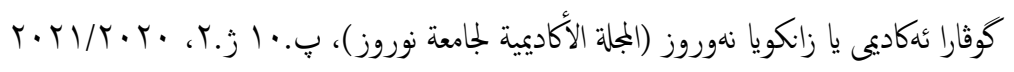

وكذلك سياسة الدخول والقرارات التي تهدف المى التحكم بالصادرات والواردات ، وما يتعلق بمعدلات الفائدة وسعر الصرف ودراسة تأثيرها على كل من الطلب على النقود والناتج القوي الإجالي وميزان المدفوعات، إذ تمارس السياسة النقدية تأثيراً غير مباشراً يتحقق عبر إجراءات تستخدما السلطة النقدية في جحم الائمان وكلفة إستخدام النقود ، ولا تحتاج الى مدة زمنية طوياة طبقاً لهيكليتها في تنفيذ أهدافها، لسرعة تأثيرها في النشاط الإقتصادي من خلال إخضاعها لقواعد عمل محددة تدفعها تلقائيا كلما تغيرت المؤشرات الاقتصادية وهذا ما يميزها عن السياسة المالية التي تمارس تأثيراً مباشراً على مستويات الدخول من خلال الثأثير على ملى المستوى الكلي في الاقتصاد بواسطة أدواتها المتثثلة في الإنفاق الحكوي والإستثاري ، وما يؤخذ عليها وجود الفجوات الزمنية أو ما يعرف بالتباطؤات ، فالسياسة النقدية هي التطبيق العملي للقوانين التي تضعها الإدارة العامة التي قد

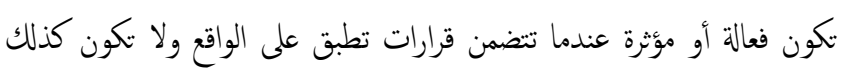
عندما تتضمن تشريعات فوقية غائبة عن التطبيق في الواقع العملي (Einziq,1972,88). للسياسة النقدية علاقة وثيقة بالنقود والجهاز المصرفي بما يتعلق بوظيفة الائتمان وكذلك بالسياسة المالية ولا سيا بالجزء المتعلق بمشكلة الدين العام ، تلك المشكلة التي ظهرت مع نهاية الحرب العالمية الثانية لتصبح

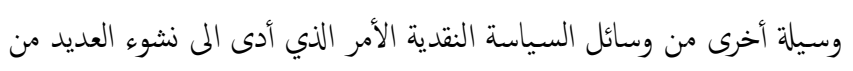
المشاكل المتعلقة بموازين مدفوعات جميع الدول التي خاضت الحرب ، وكان السبب الرئيس فيها الإنفاق الواسع للحكومات على مشاريع الإعار والإنشاء والتنمية نتيجة

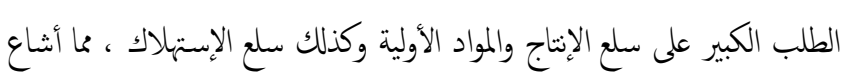
الإرباك في موازين مدفوعاتها وهذا أدى الى جعلها تفقد الكثير من أرصدتها النقدية

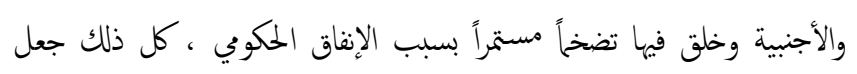
تحيق الاستقرار والتوازن في ميزان المدفوعات هدفاً أخر من أهداف السياسة النقدية ، وبذلك إتسعت وتعددت وسائل السياسة النقدية وأهدافها (السامرائي

$$
\text { والدوري ، } 2005 \text { ، 37). }
$$

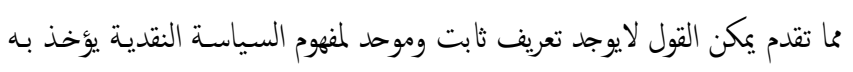
ويطبق في جميع الأحوال والظروف ، لأن الاوضاع الاقتصادية والظروف النقدية تختلف من دولة الى أخرى ، تعكس درجة التطور النقدي والمالي وغيرها من الإعتبـارات الأخــى وأثرهـا عـلى السياسـة النقديـة ، وفي معظم دول الإتحـاد

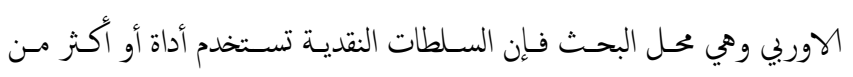
أدوات السياسـة النقديـة الكميـة الرئيسـية مثـل التغيـيـات في نسـبة الإحتياطي
في إدارة الكتلة النقدية الموحدة بإستهاف عرض النقد كهدف وسيط للثاثثير على إدارة السياسة النقدية في دول الإتحاد الأوربي وأثارها على الإقتصاد الكلي. 5.1

من أجل إختبار أثر بعض المتغيرات الاقتصادية في أداة عرض النقد ، ومن اجل بناء نموذج قياسي لتحليل هذه المتغيرات فقد تم تحديد الفترة (1995-2019 )

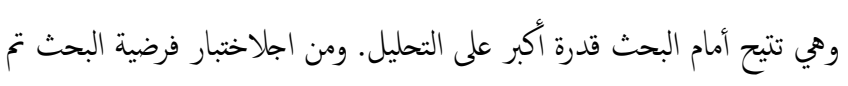
تقسيمه الى ثلاثة مباحث ، يضم الاول الاطار النظري والمفاهيمي للسياسة

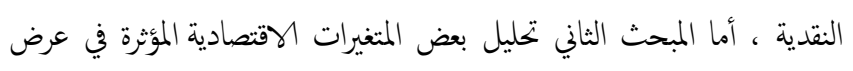
النقد. في حين جاء المبحث الثالث بعنوان قياس أثر بعض المتغيرات الاقتصادية

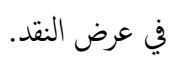

\section{2. المبحث الوول: الإطار المفاهي للسياسة الثقدية}

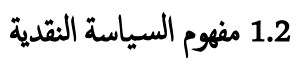

هي أداة من أدوات السياسة الإقتصادية العامة تستخدم من قبل الحكومة الى الى جانب السياسات الأخرى كالسياسة المالية وسياسة الإجور والأسعار والتجارة الخارجية للثأثير ايجابياً في مستوى النشاط الإقتصادي ، يدير السياسة النقدية في كل بلد البنك المركزي ويكون عمله مستقلاً عن عمل الحكومة ولكنه بعمل ضمن قوانين وتشريعات سنتها الحكومة ، عاكساً في الوقت نضه درجة التطور الإقتصادي والإجتاعي للبلد من خلال معرفة عناصر النظام النقدي. كما عرفت السياسة النقدية بانها إدارة التوسع والإنكاش في العرض النقدي لغرض تحقيق هدف معين ( 455 : R.P.Kent , 1972 ) في حين تعرف على أنها

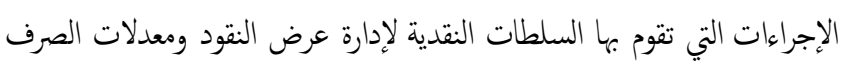
والفائدة والثأثير في قرارات الائمان لتحقيق أهداف إقتصادية محددة. أو هي عبارة عن البرامج والإجراءات التي تنهجها السلطات النقدية لتنظيم النقد في المجنع وصولا للأهداف المرغوبة ، يقوم بهذه الإجراءات البنك المركزي Christine ) Ammer and S.Ammer, 1977, 269). لقد تغير مفهوم السياسة النقدية من المحدود لآلية عمل السياسة النقدية الى التفسير الشامل الذي يضم جميع القرارات والإجراءات المدروسة التي تتخذ من قبل السلطة النقدية للثأثير على حجم النقود من حيث قيمتها وكلفة توفيرها بوسائل مباشرة وغير مباشرة . وكذلك إتساع مساحة عمل أهدافها لتشمل أهداف نقدية وغير نقدية مثل الرقابة على الأسعار والإجور والفعاليات الخاصة بالميزانية ، 


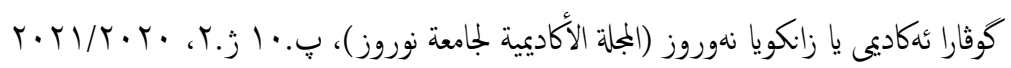

إنها تؤدي إلى زيادة التحصيلات ، أما الواردات فنعد في الجانب المدين من ميزان المدفوعات باعتبارها بنوداً سـالبة إذ يترتب عليها مدفوعات للخارج. حيث تعمل السياسة النقدية على تحسين وضع ميزان المدفوعات من خلال التحكي بقيمة النقود فعندما تعاني من جز في ميزان مدفوعاتها تحاول تخفيض قيمة العملة المحلية من أجل تحقيق فـائض في الميزان التجـاري والتخلص مـن العجز في ميزان المدفوعات (معروف ، 2006 ، 139) بافتراض هناك مرونة عالية للقطاعات الاقتصادية

$$
\text { المساهمة في الناتج المحلي الاجلالي. }
$$

4.2.2 المسامة في تحقيق مستوى عال من الاستخدام للموارد والعلالة أن الاستخدام الأمثل هدف مرغوب فيه وإن التوظف في مستويات مقبولة يراد بذلك حرص السلطة النقدية على ابقاء النشاط الاقتصادي عند أعلى مستوى مككن مـن التوظف والعالة للموارد ، وذلك مـن خلال انتهـاج سياسـات كفيلة بتجنب الاقتصاد (البطالة) من خلال تحفيز الاستثمار عند إتباع سياسة تخفيض أسعار الفائدة وتقليص كلف الحصول على الائتمان ودع وتوفير الائتمان للقطاعات

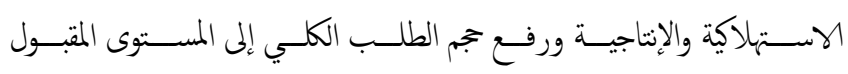

لتشغيل الموارد الإنتاجية غير المستغلة (السامرائي والدوري ، 2005 ، 189).

1.4.2.2 تشجيع النمو الاقتصادي المرغوب

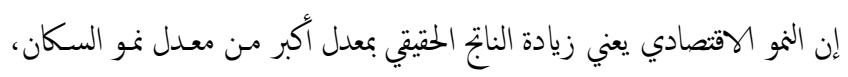

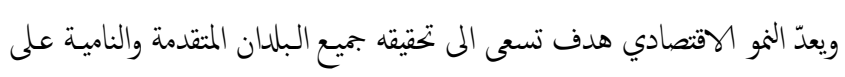
حد سواء ، من أجل إثباع حاجات الأفراد ورفع مستوى معيشتهم ورفاهيتهم

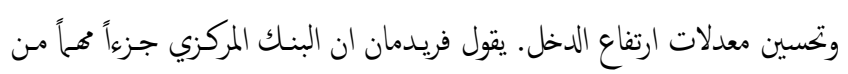

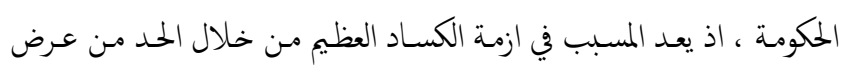
النقود ، كما حدث في أزمة 2008 ، اذ ركزت البنوك المركزية في العالم على عرض النقد وحسب راي فريدمان فان الاقتصادات سوف تعود الى حالة التشغيل الكلمـل للمـوارد ولكـن حسدث العكس ، الا ان النــــ الاقتصـادي في اوربا والولايات المتحدة واليـابان اتجه نحو الانكماش (أبو شرارة ، 2010 ، 215). ويؤكد المختصـون في البنـك المركزي الووربي على ضرورة تقيــــــــل البنـوك المركزية ، التي ينبني ان تزيد عرض النقد بمعدل سنوي ثابت وحينا تفشل هذه النظرية في تثثيت الاسعار يعمد المى زيادة او تخفيض عرض النقود وفقا لمعدل التضخم. بالاضافة الى بمموعة الادوات الكيمة المعروفة ، فالبنوك المركزية عليها

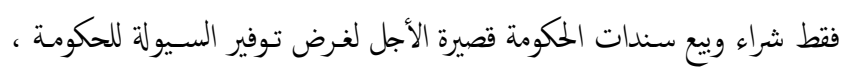
قاوم الاعضاء الاملان في البنك المركزي الووربي شراء السندات طوياة الاجل
النقدي القانوني ، والنغييرات في سعر إعادة الخصم أو سعر البنك الرسمي وسعر

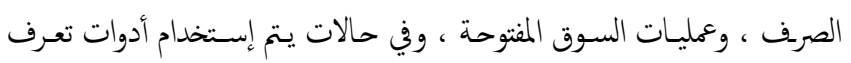

$$
\text { بأدوات الرقابة المباشرة أو الأدوات النوعية. }
$$

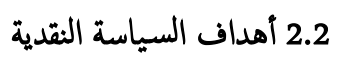
للسياسة النقدية بمموعة من الأهـداف التي تسعى لتحتيقهـا مـن خلال أدواتهـا

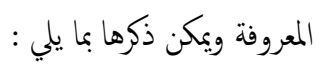

\subsection{2 تحقيق الإستقرار في مستوى الأسعار}

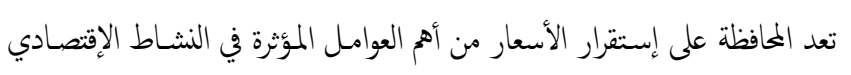
وفي المؤشرات الإقتصادية الرئيسة ، وتنحصر- في العمل على موابهـة التغـيرات المستمرة في مستوى الأسعار ، لأن أي تغير في مستويات الأسعار نخو الإرتفاع سيؤثر سلباً في قيمة النقود ويؤدي المى إنخاض القوة الشرائية لها تاركة آثار ضارة على مستوى الدخل والثروات وتخصيص الموارد الاقتصادية وبالتالي على الأداء الاقتصادي (خليل ، 1982 ، 132). 2.2.2 تحقيق الإستقرار في سعر الصرف ،

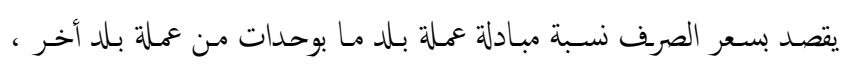
ويقصد بالصرف الأجنبي هي العملية التي بمتضاها يتم الحصول في دولة ما على سلى وسائل تسوية المدفوعات في بلد آخر (يونس ، 1984 ، 161) وبهذا المعنى فيان المقصود بالصرف هو التبادل بين العملات بهدف تحقيق التسويات والمدفوعات الدولية ، ويعد هدف تحقيق استقرار أسعار الصرف مرتبطاً بالهدف الأول وهو

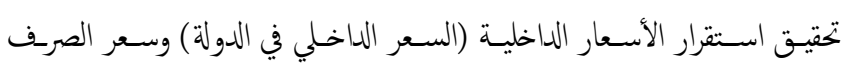
(السعر الخـارجي في الدولة) أي السعر الذي يـربط بـين الاقتصـاديات المحليـة

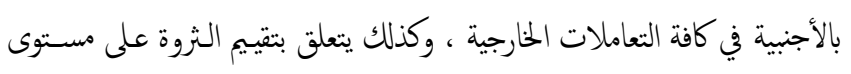
الفرد والدولة بالاضافة الم تقييم ادوات الاستثار في الاسواق المالية.

\subsection{2 العمل على تحقيق التوازن في ميزان المدفوعات} هو بيان يوضح فيه قيمة جميع السلع والخدمات والهبات والمساعدات وجميع قروض سترون رؤوس الأموال والذهـب والاحتياطات الدولية التي تأتي من الحارج إلى داخل الدولة ، أو التي تخرج من داخل الدولة إلى المنارج (خليل ، 1982 ، 749 ). وبتعبير أدق هو حساب يلخص كل المعاملات الاقتصادية بين القطاع العائلي والمنشآت والقطاع الحكوي لدولة معينة خلال مدة زمنية معينة ، مع سائر العالم الخارجي وتشمل المعاملات الصادرات والواردات والتدفقات الخختلفة لرأس المال ، وتعد الصادرات في الجانب الدائن من ميزان المدفوعات باعتبارها بنوداً موجبة إذ 


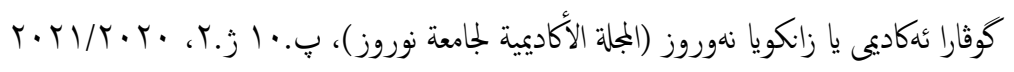

التقلبات السعرية تمانياً مع النظرية النقدية (لفريدمان) التي تقضي_ بالحفاظ على

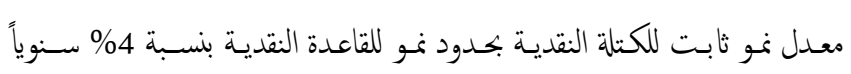
(ستيغلز ، 2020 ، 117). في ظل تخرير أسعار الصرف يصبح التحكم بالأساس النقدي كهدف وسـيط تستطيع السـلطة النقدية المحافظة على الاسـتقرار في المستوى العام للأسعار من خلال توجيه أدواته كسعر الفائدة لعلاقته بمستويات التضخم في الأجل الطويل ، أذ يصبح التحكي بنمو القاعدة النقدية هي العنصرالأكثر فاعلية في تحتيق الاستقرار للأسعار ، وتأتي الخبرة المتزاكة للساطة النقدية بفاعلية أدوات تلك المجملات وطبيعة القطاع النقدي والمصرفي أساس نجاحما (ابو شرار ، 2010 ، 186) من خلال اسعار الفائدة. نتيجة للتطورات السريعة في

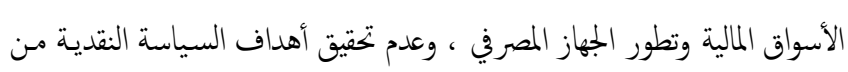
خلال الأداة السابقة اتجهت معظم البلدان المتقدمة إلى أداة سعر الفائدة قصيرة الأجل التي تستجيب للتغيرات في التضخم والناتج واستخدامها هدفاً وسيطاً.ويتم ذلك عن طريتق رفع الفائـدة قصـيرة الأجل بين المصـارف عندـ ارتفـاع معـدل

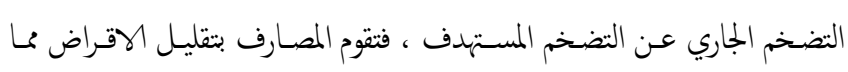
يؤدي إلى تقليص الائتمان وتقليل السيولة ومن ثم السيطرة على التضخم وتختيق الاستقرار في الأسعار (whitehead , 2003 (w).

\section{3. المبحث الثاني : تحليل بعض المثغيرات الاقتصادية الموثزة في عرض النقد:}

\section{3 مثغير عرض النقد اليورو}

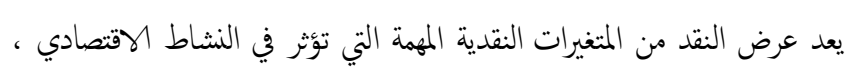
ولايشتمل عرض النقد الاوربي على المعنى الضيق فسب وانما على عرض النقد بلمعنى الواسع بسبب تطور النظام المصرفي إضافة الى وجود ثقافة مصرفية لدى الافراد وحسب راي (أنا سكوارتز وفريدمان) ان عرض النقد بالمنى الضيق لايمثل كية النقود الفعلية داخل الاقتصاد وانما عرض النقد الواسع يكون اكثر تثيلاً لكمية النقود داخل الاقتصاد لاسيا في الدول المتقدمة.

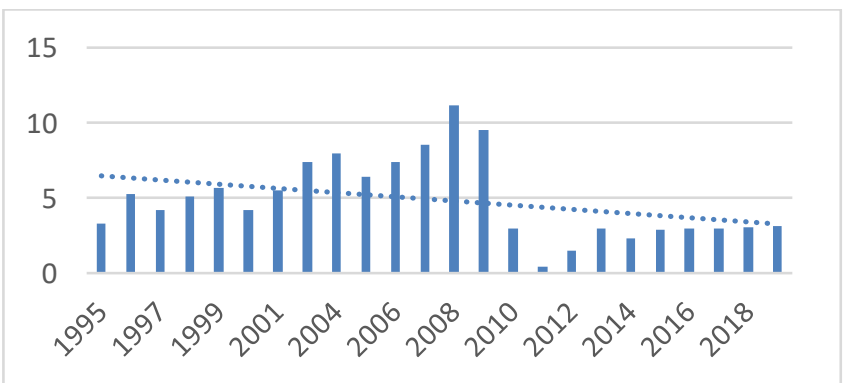

\footnotetext{
المصدر : من اعداد الباحثان بالاعتاد على ننائج البرنامج الاحصائي Excel.

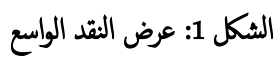

والتي ربما يكون بعضها ليس سندات حكومية. كما اضعفت القيود المفروضة بشدة من امكانية البنك المركزي الاوربي على فعله بواسطة التفويض بالتضخم وكانت

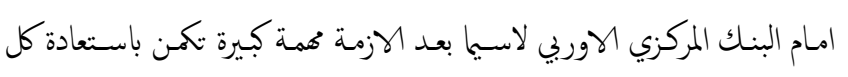
بلدان المنطقة للتشغيل الكامل (معروف ، 2006 ، 158). مـل الاقتقار الى

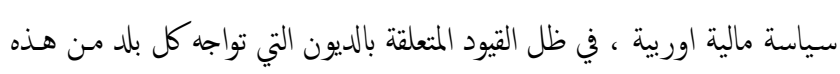
البلدان عبئا ثقيلا على السياسة النقدية وحتى في ظل عدم وجود قيود كانت قدرة البنك المركزي الووربي على الحفاظ على النمو والتشتيل الكمامل امتداد منطقة اليورو محدودة وقد ركز الاقتصاديون المحافظون الذين هيمنوا على عمل

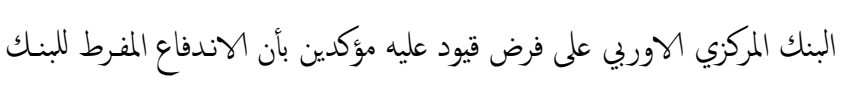
وما يككن ان يؤدي اليه من تضخم سيسبب تضخم اقتصادي ويقلل من الاستثخار

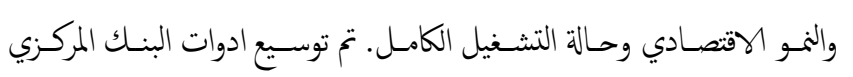

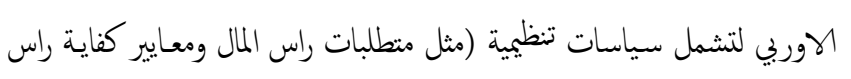
المال للبنوك) المستخدمة في صنع سياسة مالية تؤثر في اقتصادات دول الاتحاد

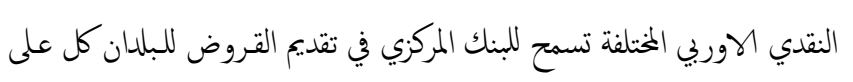
انفراد بطرق مختلفة لان سعر الفائدة مختلف على امتداد منطقة اليورو ، اقتراض

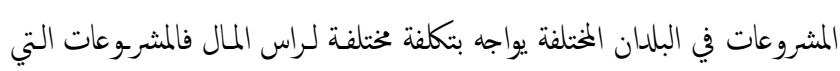

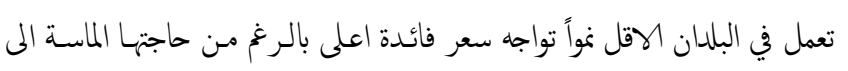

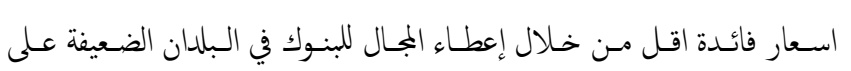
الاقراض اكثر (السامرائي والدوري ، 2005 ، 42).

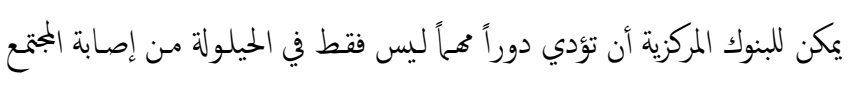
باضرار الخارجيات السلبية مثل اسـاءة استعال بطاقات الائتمان ، والافراط في تهري المخاطرة وانمـا في المسـاعدة على منح الائتمان للمشروعات الصغيرة مثثلا. تعتبر أهداف السياسة النقدية أهدافا بعيدة المدى في تأثيرها على المستوى الاقتصادي بشكل عام ، يعني أن استخدام أدوات السياسة النقدية لا يظهر بصورة سريعة

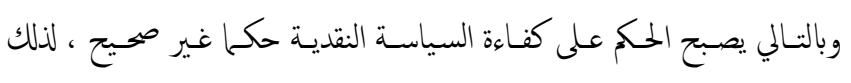
تسعى السلطة النقدية للنأثير في أهداف وسيطة تتميز باستجابتها السريعة لأدوات

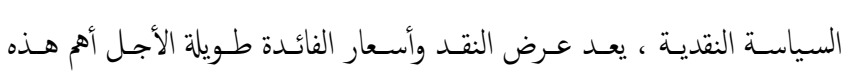

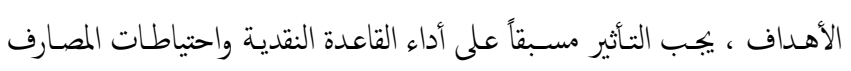
الكلية التي تعتبر جزء من القاعدة النقدية أو مـا يسمى بأهداف الأداء عبر أداة عرض النقد ، حيث أنبعت السلطات النقدية في الدول المتقدمة والنامية خلال فترة السبعينات والثزانينات أهدافأ وسيطة ترنكز على نمو الكتلاة النقدية لمواجهة 


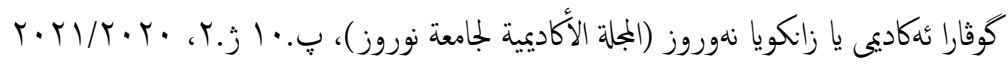

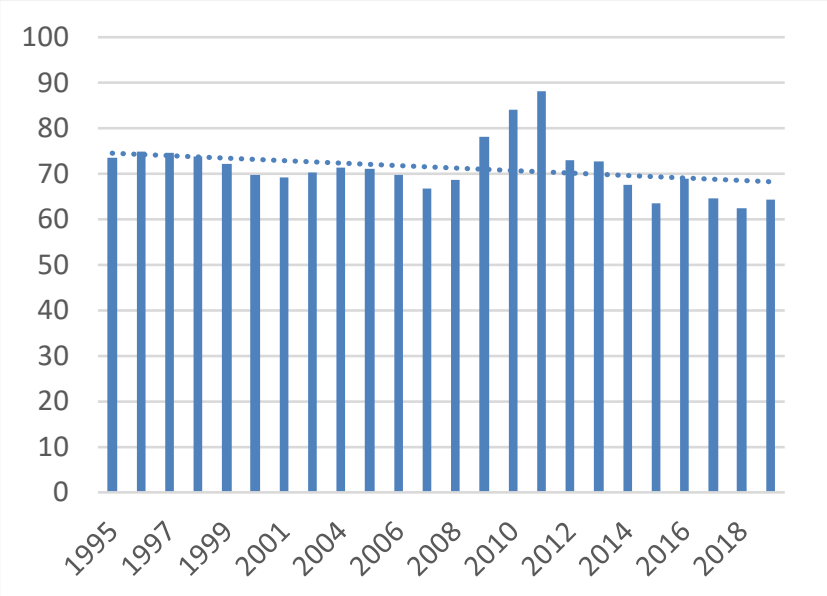

المصدر: من إعداد.الباحثان بالإعتاد.على نتائج البرنامج.الإحصائي Excel. الشكل 2: المديونية لدول الهتحاد الأوريي

حسب نتاجُج معاهدة ماسترخت فأن الدولة الراغبة في الانضام الى الاتحاد النقدي يجب ان لايتجاوز الدين العام الى الناتج المحلي الاجلالي (60\%) لكي يتسنى للدولة ان تحقق تقارباً مع الدول الاخرى في الاتحاد وبالتالي تحقق نواً

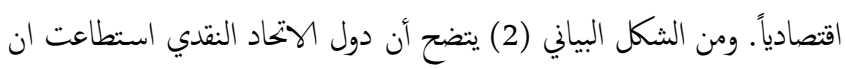
تحقق الخفاض في نسبة الدين الى الناجت الحملي الإجالي يظهر ذلك من خلال خط الاتجاه العام الذي يتجه نخو الانخفاض المتدرج يعكس الاثر الايجابي للاتحاد النقدي على الدين العام ، ال ان هناك زيادة في نسبة الدين للفترة (20082013) وهذا يعود الى الازمة المالية العالمية بالاضافة الى الكساد الذي ساد في تلك الفتزة وانخفاض اسعار النفط. مما تقدم يتضح ان الاتحاد النقدي استطاع ان يحقق تقاربا في المعيار المذكور، لان البديل الوحيد امام دول الاتحاد اعادة هيكة الديون بسبب سحب صلاحيات السياسة النقدية منها.

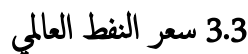

تعرض أسعار النفط والطاقة الى تقلبات في الاسعار بسبب العديد من العوامل المتداخلة الاقتصادية والسياسية والطبيعة الأمر الذي يجعل هذا المتغير الاستراتيجي غير المستقر يجعل من اسعار المنتجات الصناعية المعتمة على النفط غير مستقرة ، فني تشرين الاول 1995 تجاوز سعر البرميل خلال فترة قصيرة 40 دولار ليتجاوز هذا السعر ابان حرب الخليج الاولى ، ليستمر في الزيادة المندرجة البسيطة لغاية 2005 حيث ارتغع سعر برميل النفط بشكل لافت وصل الى 70 دولار ويعود هذا الارتفاع الى اعصار كاترينا الذي ضرب خليج المكسيك والشكل البياني (3) يوضح ذلك
ومن خلال الشكل البياني أعلاه لدول الاتحاد النقدي الاوربي يظهر ان النظام الملالي يعاني من تقلبات في عرض النقد والائتمان بسبب ضعف الضوابط التنظيمية ما يفسح المجال امام حدوث حالات من الازدهار والكساد والتضخم وهذا ما يتسم به النظام الرأسلالي بشكل عام ، حيث يوضح الشكل البياني ان هناك انخفاض واضح في سنة (2000-2001) وهذا يعود المى حالة الكساد التي مر بها العالم بسبب أحداث مبنى التجارة العالمي ، ثم اخذ الشكل البياني في الزيادة لاسيا في سنة (2008-2009) وهذا يعود الى الازمة المالية العالمية التي ظهرت انذاك بالاضافة الى الارتفاع الشديد في اسعار النفط ادى الى ظهور حالة من التضخم والزيادة في عرض النقد التي تؤدي الى الزيادة في الطلب بمقدار اكبر من قدرة العرض الكلي على توفير السلع والخدمات مما يؤدي المى الارتفاع المستمر في المستوى العام للاسعار.كما زادت البنوك فعالية عرض النقد عن طريق الزيادة في عرض الائتان اذ يضطلع البنك المركزي عملية تنظيم خلق النقود والائتمان وعادة ما يكون بشكل غير مباشر ومن المفترض ان تستطيع البنوك ضح الكمية الصحيحة من اجل وصول الاقتصاد الى حالة من الاعتدال اي لايعاني من كساد او تضخم. وقد عانى الاتحاد النقدي الاوربي بحالة من الكساد سنة 2011 ولكن سرعان ما استعاد عافيته واستمر بالنمو بشكل متزايد وبوتيرة متدرجة ، وقد اظهر خط الاتجاه العام أن هناك إنخاض بسيط في عرض النقد يميل الى الاستقرار في السنوات الاخيرة يظهر نمو واستقرارية الاتحاد النقدي الاوربي.

\section{3 المديونية المى الناتج المحلي الاججالي لدول الاتحاد الثقدي الأوريي} يعد الدين المرتفع اداة خانقة لنمو البلد هناك ثلاثة سبل تتمدها البلدان للتعامل مع ارتفاع معدلات الديون للناتج المحلي الاجالي قبل الدخول في الاتحاد النقدي الاوربي متثلة بالتضخم من أجل تخفيض القيمة الحتيقية للديون لاسيا طويلة الاجل ، وكذلك السبيل الثاني معدلات النمو في الاقتصاد فعند ارتفاع الناتج المحلي الإجالي تنخفض نسبة الديون الى الناتج المحلي الإجالي لتصبح الديون هامشية وغير همة اي لايتجاوز اجالي الدين 60\% الى الناجت المحلي الاجلالي المنصوص عليها عالمياً. ليبقى السبيل الاخير امام الاتحاد النقدي متثثلة ببرامج التقشف التي تعمل على جعل مستويات الديون أقل استدامة. 
ويظهر من الشكل البياني (4) ان صافي الميزان التجاري بدأ متزايدا محققاً فائضاً ملحوظاً ولكن سرعان ما تغير نحو العجز في سنة (2001-2002) هذا العجز يعود الى عدم الاستقرار العالمي اذ ساد العالم حالة من الركود بسبب احداث الحادي عشر من سبتمبر ايلول (استهداف مبنى التجارة العالمي) ، ليرتفع مرة اخرى وبشكل متزايد لغاية الازمة المالية العالمية التي ظهرت سنة (2008) واستمر الميزان التجاري في حالة فائض لغاية سنة (2010) ثم بدأ متذبذباً تارة بالارتفاع واخرى بالانخفاض لغاية (2015) بسبب عدم الاستقرار العلمي والهجرة الى اوربا بغعل داعش ، اخذاً فيا بعد بالارتفاع المتدرج وقد اظهر خط الاتجاه العام هناك إنخفاض في صافي الفائض في الميزان التجاري رغز توفير الاتحاد النقدي

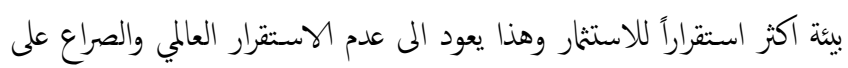
منابع الطاقة في دول الخليج وظهور المليشيات المتطرفة. 4. المبحث الثالث: قياس أثر بعض المثغيرات الإقتصادية في عرض الثقد لدول

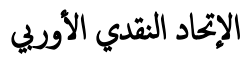
قبل الدخول في الإختبارات القياسية التي تبدأ بإختبار جذر الوحدة لابد من التعرف على متغيرات الدراسة ومعرفة علاقة المتغيرات المستقلة بالمتغير التابع وفق

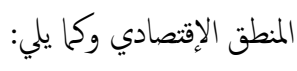
= عرض النقد الواسع في الإتحاد النقدي الاوربي. Y

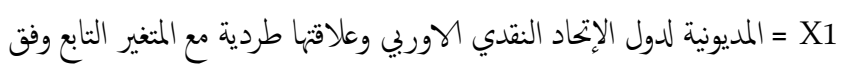
المنطق الاقتصادي. سعر النفط العالمي بالدولار وعلاقته طردية مع المتغير التابع وفق منطق X2 النظرية الاقتصادية. X3 = صافي الميزان التجاري وعلاقته طردية مع المتغير النابع وفق منطق النظرية الاقتصادية.

\section{4 إخبارات جذور الوحدة لمثغيرات الدراسة}

لتحديد رتبة التكلمل المشترك (degree of Integration) للمتغيرات سيتم الإعتاد على إختبار جذر الوحدة وسنقوم بأكثر من إختبار اللتحقق من درجة

$$
\text { ككمل متغيرات الدراسة وكما يلي: (عبد القادر، } 2007 \text { ،3). }
$$

\section{4 إختبار (دكي- فوللر) الموس(ADF Test)}

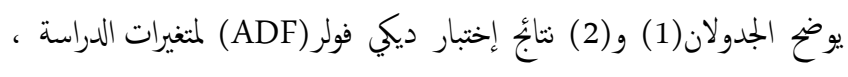
وبتطبيق خطوات الإختبار على جميع المتغيرات يمكن معرفة درجة نكاملها ، فيثلاً بالنسبة لإختبار (ADF) لسلسلة بيانات لعرض النقد في دول الإتحاد النقدي

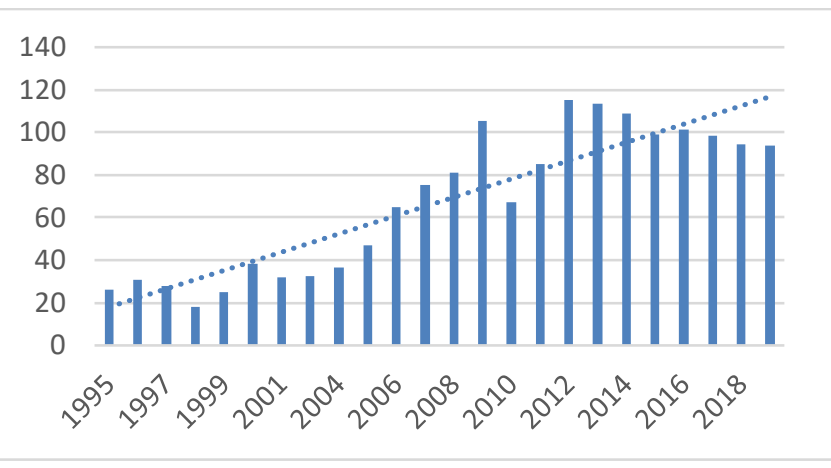

المصدر: من إعداد الباحثان بالإعتماد على نتائج البرنامج.الإحصائي Excel. الشكل 3: سعر النفط العلمي

وفي سنة 2007 ارتغع سعر برميل النفط الى ما يقارب (80) دولار واستمر في الزيادة بسبب النمو الاقتصادي العالي المتسارع وزيادة الطلب على النفط خلق حالة من الرفاه الاقتصادي العالمي حتى وصل الى (116) دولار سنة 2013 هذه الزيادة المتسارعة في اسعار النفط جعل الاتحاد النقدي الاوربي يثأثر بها الارتفاع حيث زاد من معدلات التضخم وخفض من معدلات النمو ، ولكن سرعان ما بدأ سعر النفط بالاخخاض بسبب حالة من الركود التي مر بها العالم قلل من الطلب العالمي على النفط ، ثم عاد مرة اخرى بالارتفاع البسيط ليتجاوز (70) دولار بسبب ظهور تنظيم داعش واحتلاله سوريا والعراق وتهديده لمنابع النفط في العراق · ومن خلال الشكل اعلاه يظهر خط الاتجاه العام بشكل متزايد.

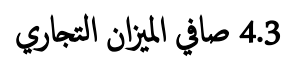

حقق الإتحاد النقدي الأوربي بيئة إقتصادية مستقرة جاذبة للإستثار الأجنبي المباشر من دول الاتحاد النقدي والدول الووربية الراغبة في الدخول مثل دول شرق ووسط اوربا التي كانت راغبة في الدخول الى السوق الاوربية المشتركة من اجل تحقيق منافع اقتصادية.

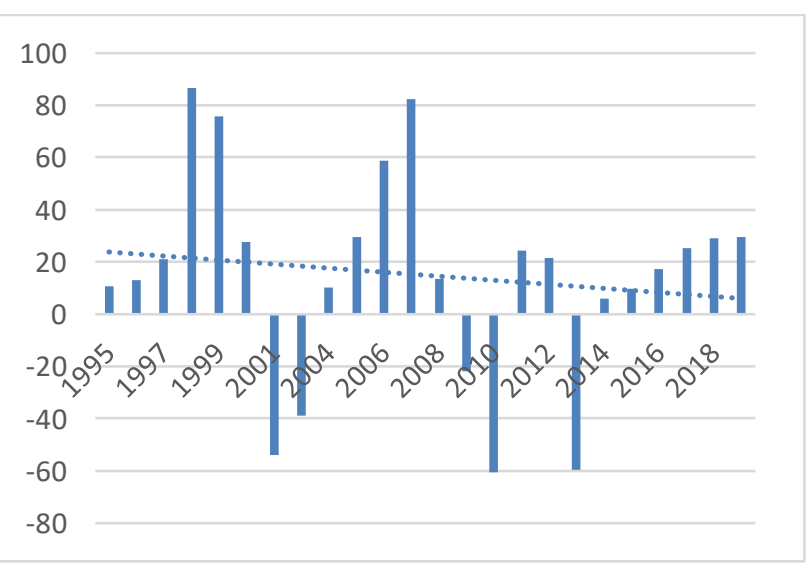

المصدر : من إعداد الباحثان بالإعتماد على نتائُ البرنامج الإحصائي .Excel. الشكل 4: صافي الميزان التجاري لدول الهتحاد 


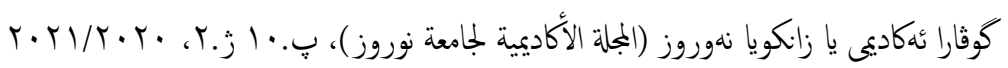

على جذر الوحدة ، أي أن المعلمتين تختلفان إحصائياً عن الصفر وبالتالي فإن الإنموذج الملائ لتمثيل بيانات السلسلة هو الأثموذج الذي يجتوي على ثابت وإتجاه ولا حاجة لإختبار أنموذج أخر أما إحصائية (pp) فنجد أنها أقل من القيم الجدولية عند مستوى(1\% و5\%) وبالتالي نتبل الفرضية القائلة بوجود جذور للوحدة عند هذين المستويين ، ومن ثخ نقوم بتطبيق إختبار(pp) مرة أخرى لتحديد الرتبة التي تستقر عندها السلاسل الزمنية وبتكرار الإختبار وأخذ الفرق الأول للسلاسل نلاحظ أن القيمة المحتسبة لإختبار (pp) أكبر من الجدولية عند المستوى (5\%و\%) ، أي أن السلسلة تستقر بعد أخذ الفرق الأول لها وبذلك

نستطيع القول بان السلسلة متكمالة من الرتبة الأولى (1) كما في الجدول (2).

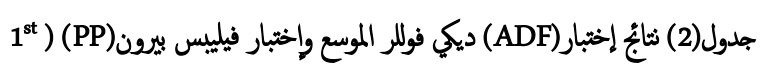
difference test

\begin{tabular}{|c|c|c|c|}
\hline PP Test & & ADF Test & Var \\
\hline None Intercept \& Individual & None & Intercept \& Individual & \\
\hline Trend Intercept & & Trend Intercept & \\
\hline
\end{tabular}

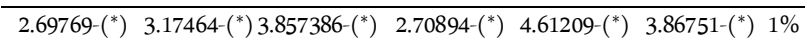

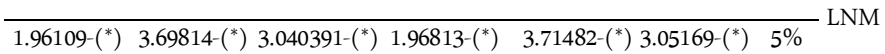
\begin{tabular}{lllll}
\hline $3.183037-$ & $4.571559-3.113180-$ & $3.995221-$ & $4.163043-$ & $3.864844-\mathrm{T}-$ statistic
\end{tabular}

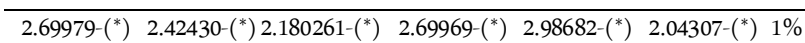

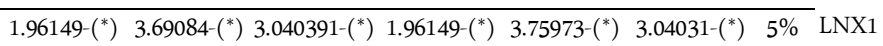
2.118071- 4.571559- 3.857386- $1.996618-\quad 4.728363-\quad 3.857386-\mathrm{T}$ - statistic

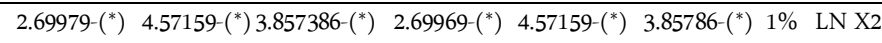

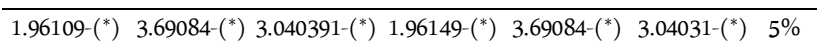
\begin{tabular}{llllll}
\hline $4.617295-$ & $6.542833-6.429595-$ & $4.578600-$ & $4.640674-$ & $4.798032-\mathrm{T}-$ statistic
\end{tabular}

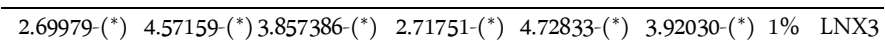

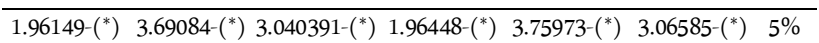
\begin{tabular}{llllll}
\hline $6.445243-$ & $13.30503-$ & $6.816662-$ & $4.395655-$ & $4.612109-$ & $4.478904-\mathrm{T}-$ statistic
\end{tabular}

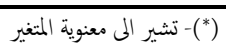
المصدر : من أعداد الباحثان بالاعتماد نتأُج البرنامج الاحصائي Eviews9

\section{- إختبار النكامل المشترك لمتغيرات الدراسة :}

بعد إختبار جذر الوحدة والتي أوضحت ان كل متغير من متغيرات الدراسة متكامل من الدرجة الأولى ، أي إن المتغيرات ساكنة عند الفرق الأول، إذ أثنار (Engle-Granger(1987)) علاقة توازن طويلة الأجل ، وبذلك يكن تمثيلها بنموذج الإرتباط الناتي وتوزيع الإبطاء الزمني ((ARDL)) Autoregressive Distributed Lag Model ( والذي يعزز إمكانية إختيار وتقدير العلاقة في المدى القصير والطويل بين متغيرات الدراسة وتجاوز الإنحدار الزائف (Spurious Regression) ، أما أذا كانت النتأجُ تدل على عدم وجود تكامل مشترك بين المنغيرات فالإنموذج المقتزح عندها للتقدير هو (VAR) ، يحتاج هذا الإختبار الى إختيار مدة الإبطاء المناسبة قبل
الأوربي ، والمديونية ، وسعر النفط الخام ، وصافي الميزان النجاري، وبعد تطبيق الإختبار تبين أن المتغيرات غير مستقرة في المستوى (level) كما أن الأثوذج لا

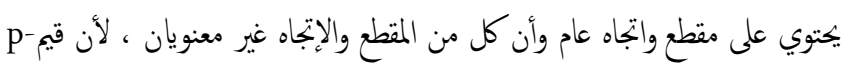
(value) المتغيرات غير مستقر ، ولم تكن إحصائية (دكي- فوللر) معنوية اذ ظهرت القيمة المتسبة أقل من الجدولية عند مستوى(1\%) و(5\%) وبالتالي لايمكن رفض فرضية العدم بمعنى السلاسل تختوي على جذر الوحدة ، وبإعادة الإختبار مرة أخرى وبأخذ الفرق الأول لمتغيرات الأموذج تبين أنها تحتوي على مقطع وإتجاه وبدون مقطع وإتجاه وعليه فإن إحصائية (ADF) المتسبة اكبر من القيمة الجدولية عند مستوى معنوية (1\%) و(5\%) يعني ذلك أن السلسلة متكمالة من الدرجة الأولى(I) ، لذلك يككن تلخيص النتائُ التي حصلنا عليها من البرنامج الإحصائي(Eviews) لإختبار(ADF) لبقية المتغيرات كما في الجدولان . (2) $(1)$

\section{(Level Test) (PP) ديكي فوللر وإختبار فيليس بيرون (ADF) نتاجُ إختبارن}

\begin{tabular}{|c|c|c|c|c|c|}
\hline \multicolumn{3}{|c|}{ PP Test } & \multicolumn{2}{|r|}{ ADF Test } & \multirow[t]{3}{*}{ Var } \\
\hline \multirow[t]{2}{*}{ None } & Intercept & Individual & None & Intercept Individual & \\
\hline & \& Trend & Intercept & & \& Trend Intercept & \\
\hline 2.692358 & 4.532598 & 3.831511- & $2.708094-$ & $4.571559-\quad 3.85786-$ & \multirow{2}{*}{ LNM } \\
\hline $1.960171-$ & $3.673616-$ & $3.029970-$ & $1.962813-$ & 3.690814- 3.04091- & \\
\hline $0.856404-$ & 1.851549 & $1.747762-$ & $0.599456-$ & $2.205704-$ & $\mathrm{T}$ - statistic \\
\hline $2.692358-$ & 4.532598 & $3.831511-$ & 2.699769 & $4.571559-3.857386-$ & \multirow[b]{2}{*}{ LNX1 } \\
\hline $1.960171-$ & $3.673616-$ & $3.029970-$ & $1.961409-$ & $3.690814-3.040391-$ & \\
\hline 1.051092 & $1.272957-$ & $0.227995^{-}$ & 0.481623 & $2.363352-\quad 0.959091-$ & $\mathrm{T}-$ statistic \\
\hline $2.692358-$ & 4.532598 & $3.831511-$ & 2.692358 & $4.532598-3.831511-$ & \multirow[t]{2}{*}{ LN X2 } \\
\hline $1.960171-$ & $3.673616-$ & 3.029970- & $1.960171-$ & $3.673616-3.029970-$ & \\
\hline 0.978914 & 2.788889 & $0.705578-$ & 0.518059 & $2.994626-\quad 0.908817-$ & $\mathrm{T}$ - statistic \\
\hline $2.692358-$ & 4.532598 & $3.831511-$ & $2.728252-$ & $4.532598-3.831511-$ & \multirow[t]{2}{*}{ LNX3 } \\
\hline $1.960171-$ & $3.673616-$ & $3.029970-$ & $1.966270-$ & $3.673616-3.029970-$ & \\
\hline 1.54347 & & $2.296139-$ & 2.230054 & 2.296139 & $\mathrm{~T}$ - statistic \\
\hline
\end{tabular}

المصدر : من إعداد الباحثان بالإعتماد على.نتائُ البرنامج الإحصائي Eviews9.

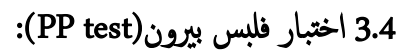

إن خطوات إختبار (فيلبس_بيرون)(pp) مشابهة لخطوات إختبار(ADF) ولتحديد رتبة التكلمل لمتغيرات الدراسة يتم الإختبار مرتين في الأولى يتم إختبار البيانات عند ما تكون السلاسل بالمستوى(level) ، ولبيانات عرض النقد والمديونية وصافي الصادرات إتضح أن كل المعلمات في الثابت والإتجاه غير معنوية أي أن(P-value) اكبر من (5\%) وبالتالي نكون ذات مستويات احصائية غير مقبولة لذلك نقبل فرضية العدم ونرفض البديلة ، وان السلاسل الزمنية تختوي 


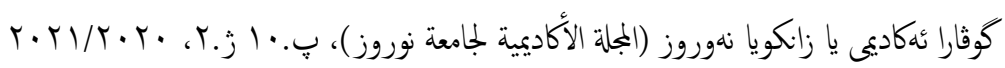

الفرضية الأولى : فرضية العدم القائلة لايوجد تكمل مشترك بين المتغيرات

$$
\text { التي تتمثل في الآتي : }
$$

$$
H_{0}: C_{1}=C_{2}=C_{3}=C_{4}=C_{5}=C_{6}=C_{7}=0
$$

الفرضية الثانية : الفرضية البديلة القائلة توجد علاقة توازنيه طويلة

$$
\text { الأجل بين المتغيرات التي تتمثل بالآتي : }
$$

$H_{1}: C_{1} \neq C_{2} \neq C_{3} \neq C_{4} \neq C_{5} \neq C_{6} \neq C_{7} \neq 0$ حيث ان التي تدخل في إختبار المعنوية المشتركة بواسطة إختبار(Wald test) الذي نحل من خلاله على إحصائية (F) المحسوبة التي يمكن مقارنتها مع (FF) الجدولية

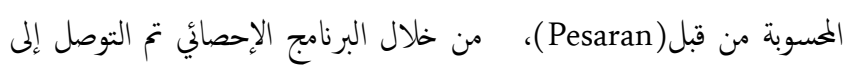
الجدول (4) الذي يبين قيمة (F) المحسوبة ، وعند المقارنة بين القيمة المحسوبة والتي مقدارها(4.63) في حالة وجود ثابت وإتجاه عام ، مع قيمة (F) الجدولية لـ (Pesaran) ، بحدهيا الأعلى والأدنى وبمستوى معنوية (0.05) ، حيث أن الحد الأدنى (2.32) والحد الأعلى(3.50) ، هذا يعني رفض فرضية العدموقبول الفرضية البديلة وبذلك نستنج بان هناك تكامل مشترك بين المتغيرات المراد إختبارها ، مما يعني وجود علاقة توازنيه طويالة الأمد بين عرض النقد في السوق الأوربية المشتركة والمديونية وسعر النفط الخام وصافي الميزان التجاري (السواعي ، (128، كما في الجدول أدناه. 2012

جدول (4) إختبار التكامل المشترك لأموذج الإتحاد الأوريي

\begin{tabular}{lcrc}
\hline \multicolumn{3}{c}{$\begin{array}{c}\text { Wald Test: } \\
\text { Equation: Untitled }\end{array}$} \\
\hline Test Statistic & Value & $\mathrm{df}$ & Probability \\
\hline F- statistic & 4.639634 & $(7,24)$ & 0.0148 \\
\hline Chi-square & 32.47744 & 7 & 0.0000 \\
\hline Null Hypothesis Summary: & & \\
\hline Normalized Restriction & Value & Std. Err. \\
$(=0)$ & & \\
\hline \multicolumn{1}{c}{$\mathrm{D}(\mathrm{X} 1(-1))$} & -0.587325 & 0.141577 \\
$\mathrm{D}(\mathrm{X} 1(-2))$ & 0.219645 & 0.146107 \\
$\mathrm{D}(\mathrm{X} 2(-1))$ & 0.010720 & 0.025729 \\
$\mathrm{D}(\mathrm{X} 2(-2))$ & -0.023759 & 0.023484 \\
$\mathrm{D}(\mathrm{X} 3(-1))$ & 1.550738 & 0.676079 \\
$\mathrm{D}(\mathrm{X} 3(-2))$ & 1.672710 & 0.681086 \\
$\mathrm{C}$ & 0.199553 & 0.399031 \\
\hline
\end{tabular}

Restrictions are linear in coefficients.

لمصدر : من إعداد الباحثان.بالإعتاد على ننائج البرنامج.الإحصائي .Eviews9.
القيام بتحليل التكمل المشترك وتحليل الإندار، وقد تم عرض نتائُ الإختبار في الجدول(3) الذي يعكس أن مدة الإبطاء المثلى هي سنتان بحسب مقياس (LR) ومعيار خطأ التنبؤ النهائي (FPE) ومعيار هنان كوين(H-Q) حيث كانت إحصائية الإختبار معنوية عندمستوى معنوية (5\%) أما إحصائية اكليكي فقد أشارت إلى إن مدة الإبطاء المثلى هي فترين (2) (AIC) معلومة شوارتز إلى أن مدة الإبطاء المثلى هي (2) أيضاً ، وبما أن المدة الثانية كانت معنوية في كل المقايس فإنه سيتم إستخداهما في إختبار (Wald Test) للتكامل المشترك وكذلك في تقدير الإنموذج متجه الإنحار الذاتي. (أبراهيم وآخرون، (285، 2002

(FPE , AIC , SC , HQ) الجدول(3) تقدير فترة الابطاء وفق المعايير)

VAR Lag Order Selection Criteria

Endogenous variables: M X1 X2 X3

Exogenous variables: $\mathrm{C}$

Date: 07/09/15 Time: 04:36

Sample: 19952014

Included observations: 18

\begin{tabular}{ccccccc}
\hline Lag & LogL & LR & FPE & AIC & SC & HQ \\
\hline 0 & -185.5536 & NA & 16489.24 & 21.06151 & 21.25937 & 21.08880 \\
\hline 1 & -136.6346 & 70.66079 & 451.3736 & 17.40385 & 18.39315 & 17.54026 \\
\hline 2 & -107.7687 & $28.86592^{*}$ & $150.9941^{*}$ & $15.97430^{*}$ & $17.7554^{*}$ & $16.21984^{*}$
\end{tabular}

${ }^{*}$ indicates lag order selected by the criterion

LR: sequential modified LR test statistic (each test at 5\% level)

FPE: Final prediction error

AIC: Akaike information criterion

SC: Schwarz information criterion

HQ: Hannan-Quinn information criterion * - تشير الى فنزة الابطاء المختارة بواسطة المعايير

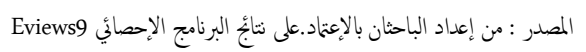
Co-Integration Test 5.4 يمثل التكامل المشترك التعبير الإحصائي لعلاقة توازنيه طوية الأجل ، يعني ذلك هلك أن كل أو بعض السلاسل الزمنية تتصف بخاصية التكمل المشترك ، فتكون العلاقات فيا بنها تتجه لتحقيق التوازن في الأمد الطويل ، بالرغ من إمكانية وجود إنرافات عن هذا الإتجاه في الأجل القصير ، وتنعكس هذه الإخرافات في البواقي التي يمثلها الحد العشوائي ، وفقاً لهذا المنطق فإن الأثموذج يكون في وضع توازن (النكامل المشترك) عندما تكون(U) مشترك أو علاقة توازنيه طوياة الأجل ، ويعتمد إختبار(Co-Integration) على 


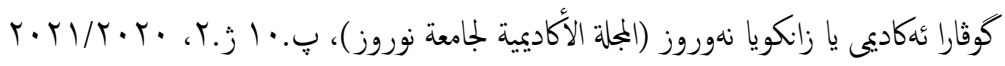

الإختبار تأثيرها العكسي يثت منطق النظرية الاقتصادية يعني أن الزيادة في المديونية بعقدار وحدة واحدة يؤدي الى تخفيض عرض النقد بمقدار المرونة البالغة (0.58) وعندما تكون متباطئة بمدين فأن تأثيره العكسي سينخفض الى (0.21). إن إخخاض تأثير نسبة الدين الى الناتج المحلي الإجالي مع الزمن يعود الى إعتماد السياسة النقدية والمالية إجراءات لتقليل الاثار السلبية على الاقتصاد. أما المتغير (2) D(X1(-2) المؤثر في عرض النقد هو سعر النفط العالمي المتباطئ زمنياً بمدتين وأظهر تأثيراً طردياً هذا يعني ان الزيادة في سعر النفط بعقدار

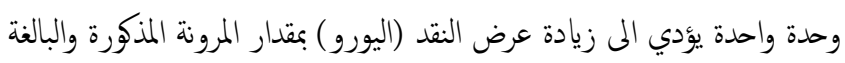
(0.2) ، وهذه حالة طبيعية تعتبر من خصائص السوق الاوربية الصناعية التي تعتمد على استيراد النفط الخام بدرجة كبيرة. في هين أثبت المثغير الفائض أو العجز في الميزان التجاري معنويته أيضاً وبعلاقة طردية تتفق مع منطق النظرية الاقتصادية وخلال مدة إبطاء واحدة ، يؤكد أن الزيادة في الصادرات تؤدي الى الزيادة في عرض النقد بمقدار المرونة المذكورة والبالغة (1.55) فالزيادة في معدلات النمو الحقيقي تجعل البنك المركزي الأوربي قادر على إصدار العملة اليورو دون تأثيره على مستوى الأسعار كما أن هذا النمو يحقق زيادة في الصادرات وبالتالي يحقق فائضاً في ميزان المدفوعات. أما تأثير حالة الميزان التجاري في الفرق الثاني فقد أظهر تأثيراً طردياً بمقدار المرونة البالغة

من خلال ما تقدم يظهر لنا ان متغيرات الدراسة ذات تاثير معنوي في عرض النقد الووربي وهذا يثبت عكس الفرضية التي انطلق منها البحث.

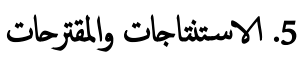

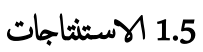

يعد عرض النقد الواسع من المتغيرات النقدية المهمة ذات التأثير الكبير في النشاط الاقتصادي والتي تعتبر من إهتمات البنك المركزي الاوربي اذ سحب التكامل النقدي صلاحيات التحكم بعرض النقد للدول المنضوية تحته

$$
\text { وجعلها تحت تصرف البنك المركزي الاوربي. }
$$

أثبتت نتائج إختبار جذر الوحدة أن متغيرات الدراسة لم تستقر في المستوى وأستقرت في الفرق الأول ، لذلك يمكن الإعتاد عليها في الإختبار واتخاذ القرار بشأنهاكون النتائج التي نتوصل لها حقيقية وغير مزيفة.

$$
\text { تقدير معادلة الإنحدار لعرض الثقد في الاتحاد النقدي الاوري: }
$$

تم تقدير معلمات المتغيرات للأثموج القياسي المستخدم في الأجل الطويل، أذ قمنا بإختبار الدالة ذات التقدير المتغيرات الأكثر معنوية كما يلي في صورته الكاملة : جدول (5) مقدرات معادلة عرض الثقد في الأجل الطويل على وفق أثموذج) (ARDL)

Dependent Variable: $\mathrm{D}(\mathrm{M})$

\section{Method: Least Squares}

Date: 07/09/15 Time: 04:43

Sample (adjusted): 19982014

\begin{tabular}{|c|c|c|c|c|}
\hline Variable & Coefficient S & Std. Error & t-Statistic & Prob. \\
\hline $\mathrm{D}(\mathrm{X} 1(-1))$ & -0.587325 & 0.141577 & -4.148437 & 0.0020 \\
\hline $\mathrm{D}(\mathrm{X} 1(-2))$ & -0.219645 & 0.146107 & -2.503314 & 0.0167 \\
\hline $\mathrm{D}(\mathrm{X} 2(-1))$ & 0.010720 & 0.025729 & 0.416639 & 0.6857 \\
\hline $\mathrm{D}(\mathrm{X} 2(-2))$ & -0.023709 & 0.023484 & -2.011721 & 0.0355 \\
\hline $\mathrm{D}(\mathrm{X} 3(-1))$ & 1.550738 & 0.676079 & 3.293722 & 0.0447 \\
\hline $\mathrm{D}(\mathrm{X} 3(-2))$ & 1.672710 & 0.681086 & 2.455946 & 0.0339 \\
\hline C & 0.199553 & 0.399031 & 0.500094 & 0.6278 \\
\hline R-squared & & 0.763619 & Mean dependent var & -0.135882 \\
\hline Adjusted R-squared & & 0.681791 & S.D. dependent var & 2.191606 \\
\hline S.E. of regression & & 1.347809 & Akaike info criterion & 3.727740 \\
\hline \multirow[t]{2}{*}{ Sum squared resid } & & 18.16590 & Schwarz criterion & 4.070827 \\
\hline & \\
\hline Log likelihood & \multicolumn{3}{|c|}{$-24.68579_{\text {criter. }}$} & 3.761843 \\
\hline F-statistic & & 5.384108 & Durbin-Watson stat & 2.438744 \\
\hline Prob(F-statistic) & & 0.010011 & & \\
\hline
\end{tabular}

Included observations: 17 after adjustments

تم إختيار الصيغة اللوغارتيمة كأفضل صيغة مثلت الدالة في الأجل الطويل، وعكست قيمة(t) المحسوبة معنوية معظم المتغيرات ، كما قدرت قيمة (R) بنحو (0.68) وهي نسبة ما فسرته المتغيرات التوضيحية في المتغير التابع المتمثل بعرض النقد الواسع في الاقتصاد الأوربي خلال مدة الدراسة ، في هين أن المتغيرات الأخرى التي لم يتضمنها الأثموذج تؤثر بنسبة (0.32) ، وتبين من (F) المحسوبة أنها معنوية للدالة بصورة عامة ، أما ما يتعلق بقيمة (D.W) والبالغة (2.4) كانت في منطقة القبول ولم تظهر مشكلة الإتباط الذاتي . إن إستخدام التحليل الإحصائي للعوامل المؤثرة في عرض النقد (اليورو) يظهر

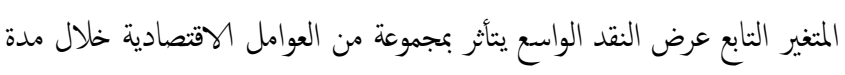
الدراسة (1995-2019) ، ويكن إدراج متغيرات الدراسة الثلاثة بما يلي : حيث أظهرت النتائُ معنوية المتغير نسبة الدين الى الناتج المحلي الإجالي D(X1(-1)) في الفرق الأول وخلال مدة إبطاء زمني سنة واحدة إذ بينت ننائج 
5. د. ساي خليل ، 1982 ، النظريات والسياسات النقدية والمالية ، الكويت ، الكناب 6. علي عبد الفتاح ابو شرار ، 2010 ، الاقتصاد الدولي نظريات وسياسات ، دار المسيرة للنشر والتوزيع والطباعة ، الطبعة الثانية. 7. الإسكندرية وبيروت. 8. د. يسري السامرائي، د. زكريا الدوري ، 2005 ، البنوك المركزية والسياسة النقدية .

2.6

1. 1-R.P.Kent, 1972, Money and BanKing, $6^{\text {th }}$ ed, insdale, IL, Rineharat and Winston, Inc.

2. P. Einziq, 1972 , Atext Book on monetary policy, $3^{\text {rd }}$. ed, London, MAGMILAN St. Martin's Press.

3. Christine Ammer and Dean S. Ammer, 1977, Dictionnary of Busness and Economy, New York; Macmillan Publishing Co.

4. D.C Vangham Whitehead ,2003, European Union Enlargement Versus Social Europe, Edward Elgar Cheltenham.
كما أثبتت نتاجُ إختبار الإبطاء الزمني أن أفضل فتزة زمنية للإبطاء هي سنتين عندها تظهر نتاجُح الإموذج أكثر معنوية من الفترات الأخرى يعني ان المتغيرات المستقلة لاتؤثر في الظاهرة المدروسة بشكل مباشر وانما تحتاج الى فترة زمنية تصل الى سنة ليظهر تأثيرها. تبين بأن هناك تكامل مشترك بين متغيرات الدراسة وبأكثر من إتجاه يعكس أن هناك علاقة طويلة الأجل تبين ذلك من خلال إختبار wald test

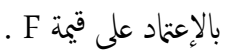
أظهرت نتائح الإنموذج القياسي معنويته من الناحية الإحصائية والقياسية ، ولم تظر مشكلة الإرتباط الذاتي وكانت قيمة معامل التحديد المصحح (688) يعني أن متغيرات الدراسة تؤثر بهذا المقدار.

2.5

العمل على زيادة التخصص ضمن دول الاتحاد النقدي والوصول الى الإنتاج الكبير من أجل التصدير وتحقيق فائض في ميزان المدفوعات.

ينبني أن تتراوح الزيادة السنوية في عرض النقد بين (3-5\%) أي حسب الزيادة في معدلات النمو الاقتصاد من أجل تفادي التضخم وتحقيق إستقرار

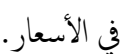

ضرورة الإعتاد على سياسة إقتصادية تقشفية من أجل تقليل نسبة الدين العام المى الناجت المحلي الإجالي وجعله ضمن الحد المنصوص عليه في معاهدة ماستريخت.

ضرورة إيجاد مصادر طاقة متجددة بتكلفة أقل من أجل تفادي الإضطرابات في أسعار النفط وتحقيق قيمة مضافة.

6. قائمة المصادر

1.6

1. السيد متولي عبد القادر ، 2007 ، اشتقاق نموذج تصحيح الخطأ من إختبار التكلمل

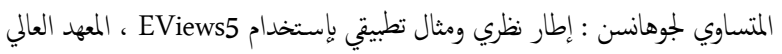

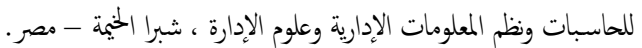

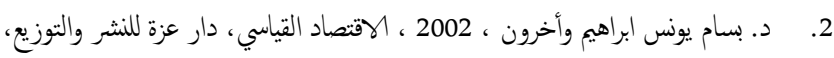
الخرطوم ، السودان. - مان. 3. د. خالد محمد السواعي ، Eviews ، والقياس الاقتصادي ، دائرة المكنبة 2012 ، المان. الوطنية ، الطبعة الاولى ، عمان الاردن.

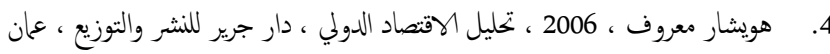

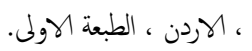




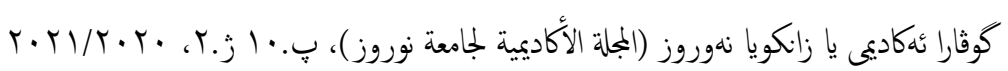

الملحق (1) عرض النقد والمديونية وسعر النفط وصافي الميزان التجاري في دول الاتحاد النقدي الأوريي

\begin{tabular}{|c|c|c|c|c|}
\hline 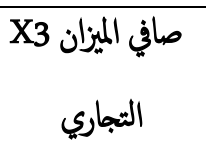 & سعر النفط العالمي X2 سع & 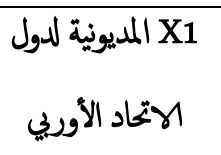 & $\begin{array}{c}\text { Y عرض النقد الواسع } \\
\text { (اليورو) }\end{array}$ & التطاعات \\
\hline 10.8 & 26.01 & 73.4 & 3.3 & 1995 \\
\hline 12.9 & 30.69 & 75 & 5.3 & 1996 \\
\hline 20.9 & 27.71 & 74.6 & 4.2 & 1997 \\
\hline 86.7 & 18.17 & 73.8 & 5.1 & 1998 \\
\hline 75.9 & 25.13 & 72.2 & 5.7 & 1999 \\
\hline 27.9 & 38.55 & 69.7 & 4.2 & 2000 \\
\hline-53.7 & 32.15 & 69.1 & 5.5 & 2001 \\
\hline-38.9 & 32.4 & 70.4 & 7.4 & 2002 \\
\hline 10 & 36.5 & 71.3 & 8 & 2004 \\
\hline 29.5 & 47.19 & 71 & 6.4 & 2005 \\
\hline 59 & 65.03 & 69.6 & 7.4 & 2006 \\
\hline 82.6 & 75.28 & 66.7 & 8.5 & 2007 \\
\hline 13.5 & 81.33 & 68.7 & 11.2 & 2008 \\
\hline-22 & 105.23 & 78.2 & 9.5 & 2009 \\
\hline-60.32 & 66.97 & 84.2 & 3 & 2010 \\
\hline 24.58 & 84.93 & 88.1 & 0.4 & 2011 \\
\hline 21.6 & 115.22 & 73.1 & 1.5 & 2012 \\
\hline-59.5 & 113.31 & 72.8 & 3 & 2013 \\
\hline 5.8 & 108.66 & 67.6 & 2.29 & 2014 \\
\hline 9.7 & 99 & 63.4 & 2.89 & 2015 \\
\hline 17.3 & 101.14 & 68.9 & 2.95 & 2016 \\
\hline 25.43 & 98.23 & 64.7 & 2.97 & 2017 \\
\hline 28.88 & 94.67 & 62.5 & 3.03 & 2018 \\
\hline 29.76 & 93.85 & 64.3 & 3.12 & 2019 \\
\hline
\end{tabular}

Sources: Eurostat. and ECB calculations.

Notes: Annual data are calculated using non-seasonally. adjusted data. Eurostat's second release of national accounts for the fourth quarter

of 2012 (which includes the expenditure breakdown) took place after the cut-off date for data for this report.

(1Percentage change compared with the same period a year earlier.

(2Percentage change compared. with the previous quarter.

(3Including acquisitions less disposals of valuables.

(4Imports and exports cover goods and services and include internal cross-border trade in the euro area. Since intra-euro area trade is not 